\title{
DISCUSSION OF CHRISTOFIDES' CONJECTURE REGARDING WANG'S PREMIUM PRINCIPLE
}

\author{
$\mathrm{BY}$ \\ VIRGINIA R. YOUNG \\ University of Wisconsin-Madison
}

\section{INTRODUCTION}

Christofides (1998) studies the proportional hazards (PH) transform of Wang (1995) and shows that for some parametric families, the PH premium principle reduces to the standard deviation (SD) premium principle. Christofides conjectures that for a parametric family of distributions with constant skewness, the PH premium principle reduces to the SD principle. I will show that this conjecture is false in general but that it is true for location-scale families and for certain other families.

Wang's premium principle has been established as a sound measure of risk in Wang $(1995,1996)$, Wang, Young, and Panjer (1997), and Wang and Young (1998). Determining when the SD premium principle is a special case of Wang's premium principle is important because it will help identify circumstances under which the more easily applied SD premium principle is a reliable measure of risk.

\section{RESULTS}

First, recall that a distortion $g$ is a non-decreasing function from $[0,1]$ onto itself. Wang's premium principle, with a fixed distortion $g$, associates the following certainty equivalent with a random variable $X$, (Wang, 1996) and (Denneberg, 1994):

$$
H_{g}[X]=\int_{-\infty}^{0}\left\{g\left[S_{X}(t)\right]-1\right\} d t+\int_{0}^{\infty} g\left[S_{X}(t)\right] d t,
$$

in which $S_{X}$ is the decumulative distribution function (ddf) of $X, S_{X}(t)=\operatorname{Pr}(X>t), t \in \mathbf{R}$. If $g$ is a power distortion, $g(p)=p^{c}$, then $H_{g}$ is the proportional hazards (PH) premium principle (Wang, 1995).

Second, recall that a location-scale family of ddf's is $\left\{S_{Z}\left(\frac{t-\mu}{\sigma}\right): \mu \in \mathbf{R}, \sigma>0\right\}$, in which $S_{Z}$ is a fixed ddf. Alternatively, if $Z$ has ddf $S_{Z}$, then $\{X=\mu+\sigma Z: \mu \in \mathbf{R}, \sigma>0\}$ forms a location-scale 
family of random variables, and the ddf of $X=\mu+\sigma Z$ is $S_{Z}\left(\frac{t-\mu}{\sigma}\right){ }^{1}$ Examples of location-scale families include the normal, Cauchy, logistic, and uniform families (Lehmann, 1991, pp. 20f). In the next proposition, I show that Wang's premium principle reduces to the SD premium principle on a location-scale family. Christofides (1998) observes this phenomenon in several special cases.

Proposition 1: Consider a location-scale family II. For a fixed distortion $g$, Wang's premium principle reduces to the standard deviation principle on $\Pi$.

Proof: For $X \in \Pi$, one has $H_{g}[X]=\mu+\sigma H_{g}[Z]$, because $H_{g}$ is scale and translation invariant (Denneberg, 1994) ${ }^{2}$. Also, the SD premium principle applied to $X$ gives the premium

$$
\mathrm{E} X+\lambda \sqrt{\operatorname{Var} X}=\mu+\sigma \mathrm{E} Z+\lambda \sigma \sqrt{\operatorname{Var} Z}=\mu+\sigma(\mathrm{E} Z+\lambda \sqrt{\operatorname{Var} Z}),
$$

for some $\lambda>0$. Equate this expression with $H_{g}[X]$ to obtain

$$
\lambda=\frac{H_{g}[Z]-\mathrm{E} Z}{\sqrt{\operatorname{Var} Z}} .
$$

Note that $\lambda$ is independent of $\mu$ and $\sigma$ from which it follows that $H_{g}$ reduces to the $\mathrm{SD}$ premium principle on $\Pi$.

The skewness of random variables in a location-scale family is constant, in which skewness is defined by

$$
\text { Skew } X=\frac{\mathrm{E}\left[(X-\mathrm{E} X)^{3}\right]}{(\operatorname{Var} X)^{\frac{3}{2}}} .
$$

Indeed, if $X=\mu+\sigma Z, \sigma>0$, then Skew $X=$ Skew $Z$. Thus, Proposition 1 demonstrates that Christofides' conjecture is true for a location-scale family of random variables.

In the next proposition, I show that Christofides' conjecture is generally true in the case of a two-parameter family of ddf's if one of the parameters is a scale parameter.

Proposition 2: Let $\Pi$ be a family of random variables whose distribution functions depend on two parameters, $\alpha$ and $\beta$, of which $\beta$ is a scale parameter. If Skew $X=c$ has a unique solution $\alpha_{0}$ for $c>0$ a constant and

The premium principles considered in this discussion depend only on the marginal ddf of a given random variable, so it is immaterial as to whether one considers a location-scale family of ddf's or random variables.

2 As an aside, this property should more rightly be called scale and translation equivariance, instead of invariance. 
for $X \in \Pi$, then for a fixed distortion $g$, Wang's premium principle reduces to the standard deviation principle on the subfamily of $\Pi$ with constant skewness $c$.

Proof: Because $\beta$ is a scale parameter, Skew $X$ is a function of $\alpha$ only. If Skew $X=c$ has a unique solution $\alpha_{0}$, then the subfamily of $I$ with constant skewness $c$ has $\alpha=\alpha_{0}$ fixed and $\beta>0$ arbitrary. On this subfamily $H_{g}[X]=\beta H_{g}\left[Z_{\left.\alpha_{0}\right]}\right]$, for some random variable $Z_{\alpha_{0}}$ whose ddf depends only on $\alpha_{0}$. The SD premium principle on the subfamily gives

$$
\mathrm{E} X+\lambda \sqrt{\operatorname{Var} X}=\beta \mathrm{E} Z_{\alpha_{0}}+\lambda \beta \sqrt{\operatorname{Var} Z_{\alpha_{0}}}=\beta\left(\mathrm{E} Z_{\alpha_{0}}+\lambda \sqrt{\operatorname{Var} Z_{\alpha_{0}}}\right),
$$

for some $\lambda>0$. Equate this expression with $H_{g}[X]$ to obtain

$$
\lambda=\frac{H_{g}\left[Z_{\alpha_{0}}\right]-\mathrm{E} Z_{\alpha_{0}}}{\sqrt{\operatorname{Var} Z_{\alpha_{0}}}}
$$

Note that $\lambda$ is independent of $\beta$ from which it follows that $H_{g}$ reduces to the SD premium principle on the subfamily of $\Pi$ with constant skewness $c$.

Of the following examples, the first two demonstrate Proposition 2, and the third examines the lognormal family. Finally, the fourth shows that Christofides' conjecture is not true in general.

\section{Examples:}

(1) Let $\Pi=\{X: X \sim \operatorname{Gamma}(\alpha, \beta), \alpha>0, \beta>0\}$, in which the probability density function of the $\operatorname{Gamma}(\alpha, \beta)$ is $\frac{\beta^{\alpha}}{\Gamma(\alpha)} x^{\alpha-1} e^{-\beta x}, x>0$. In this case, $\beta$ is a scale parameter, and Skew $X=\frac{2}{\sqrt{\alpha}}$ has a unique inverse. Thus, the conditions of Proposition 2 are satisfied, and Wang's premium principle reduces to the SD premium principle on any subfamily of $\Pi$ with constant skewness.

(2) Let $\Pi=\{X: X \sim \operatorname{Pareto}(\alpha, \beta), \alpha>3, \beta>0\}$, in which the ddf of the Pareto $(\alpha, \beta)$ is $\left(\frac{\beta}{\beta+t}\right)^{\alpha}, t>0$. In this case, $\beta$ is a scale parameter, and Skew $X=\frac{2(\alpha+1)}{\alpha-3} \sqrt{\frac{\alpha-2}{\alpha}}$ has a unique inverse. Thus, the conditions of Proposition 2 are satisfied, and Wang's premium principle reduces to the SD premium principle on any subfamily of $\Pi$ with constant skewness.

(3) Let $\Pi=\{X: X \sim \operatorname{Lognormal}(\mu, \sigma), \mu \in \mathbf{R}, \sigma>0\}$, in which the ddf of the $\operatorname{Lognormal}(\mu, \sigma)$ is $\Phi\left(\frac{\mu-\ln t}{\sigma}\right), t>0$, where $\Phi$ is the cumulative distribution function of the standard normal. In this case, $\mu$ is not a scale parameter, however, $e^{\mu}$ factors from $H_{g}[X], \mathrm{E} X$ and $\sqrt{\operatorname{Var} X}$. Also, Skew $X=\frac{e^{3 a^{2}}-3 e^{\sigma^{2}}+2}{\left(e^{\sigma^{2}}-1\right)^{\frac{3}{2}}}$ has a unique inverse. Thus, even though the 
conditions of Proposition 2 are not satisfied, Wang's premium principle reduces to the SD premium principle on any subfamily of $\Pi$ with constant skewness.

(4) Let $\Pi=\{X: X \sim$ Two-sided Exponential $(\alpha, \beta, w), \alpha>0, \beta>0$, $0<w<1\}$, in which the ddf of the Two-sided Exponential $(\alpha, \beta, w)$ is $S_{X}(t)= \begin{cases}w+(1-w)\left(1-e^{\beta t}\right), & t<0 ; \\ w e^{-\alpha t}, & t \geq 0 .\end{cases}$

Let $g$ be the power distortion given by $g(p)=p^{0.5}$. In this case, $H_{g}[X]$ has the closed form

$$
H_{g}[X]=-\frac{2}{\beta}\left[(1-\sqrt{w})-\ln \left(\frac{2}{1+\sqrt{w}}\right)\right]+\frac{2 \sqrt{w}}{\alpha} .
$$

If $\alpha_{1}=1, \beta_{1}=4$, and $w_{1}=0.9$, then Skew $X_{1}=1.84166$. Similarly, if $\alpha_{2}=1, \beta_{2}=2.27466$, and $w_{2}=0.1$, then Skew $X_{2}=1.84166=$ Skew $X_{1}$. However, $\frac{H_{g}\left[X_{1}\right]-\mathrm{E} X_{1}}{\sqrt{\operatorname{Var} X_{1}}}=0.98684$, while $\frac{H_{g}\left[X_{2}\right]-\mathrm{E} X_{2}}{\sqrt{\operatorname{Var} X_{2}}}=1.02386$. Thus, the PH premium principle does not reduce tot he SD premium principle on the subfamily of $\Pi$ with constant skewness 1.84166 .

Although Wang's premium principle reduces to the SD premium principle for various classes of random variables, Wang's premium principle with a fixed distortion produces a different " $\lambda$ " for different classes of distributions because it reflects the right tail risks of the classes. It remains an open problem to determine under what circumstances Wang's premium principle reduces to the SD principle. This is a subject of future research.

\section{ACKNOWLEDGEMENTS}

I thank Shaun Wang and Jan Dhaene for helpful comments. I also thank an anonymous referee for pointing out two errors in my original paper and for providing other useful comments.

\section{REFERENCES}

Christofides, S. (1998), Pricing for risk in financial transactions, Proceedings of the GISG/ ASTIN Joint Meeting in Glasgow, Scotland, October, 1998, 2: 63-109.

Denneberg, D. (1994), Non-Additive Measure and Integral, Kluwer, Dordrecht.

LehmanN, E.L. (1991), Theory of Point Estimation, Wadsworth, Belmont, California.

WANG, S.S. (1995), Insurance pricing and increased limits ratemaking by proportional hazards transforms, Insurance: Mathematics and Economics, 17: 43-54.

WANG, S.S. (1996), Premium calculation by transforming the layer premium density, $A S T I N$ Bulletin, 26: 71-92. 
DISCUSSION OF CHRISTOFIDES' CONJECTURE REGARDING WANG'S PREMIUM PRINCIPLE 195

WANG, S.S. and YOUNG, V.R. (1998), Ordering risks: Expected utility theory versus Yaari's dual theory of risk, Insurance: Mathematics \& Economics, 22: 145-161.

Wang, S.S., Young V.R., and PANJER H.H. (1997), Axiomatic characterization of insurance prices, Insurance: Mathematics and Economics, 21(2): 173-183.

VIRGINIA R. YOUNG

School of Business

University of Wisconsin-Madison

Madison, Wisconsin, USA, 53706 